\title{
THE VARIATION OF CASUAL, BASAL, AND SUPPLEMENTAL BLOOD PRESSURES IN HEALTH AND IN ESSENTIAL HYPERTENSION
}

\author{
BY \\ JOHN ALEXANDER KILPATRICK \\ From the Department of Medicine, University of Otago, New Zealand \\ Received December 10, 1947
}

The levels of the blood pressure as ordinarily measured are widely variable but levels observed under standard conditions have been suggested to be less variable. Conșequently, standardized procedures have been devised to give stable blood pressure levels and the results, termed basal blood pressures, have been found to be much lower than the casual pressures ordinarily obtained. No evidence has so far been presented to show that such basal pressures are physiologically constant in each individual.

The term basal blood pressure originated when Addis (1922) used the expression to describe the level he had recorded in patients before they rose from bed in the morning. He contrasted these low levels with those recorded later in the day. Addis observed a much smaller difference between daytime and basal pressures in cases of glomerulonephritis than in cases of essential hypertension. Ayman (1931) suggested means whereby more stable levels of blood pressure could be obtained especially as a preparatory step to evaluate the effects of treatment on essential hypertension, but did not refer to these levels as basal. The term basal blood pressure was used in the Joint Report of the Committees of the Cardiac Society of Great Britain and Ireland and the American Heart Association on the standardization of methods of measuring the arterial blood pressure (1938). This committee stated: "In detailed researches on blood pressure the use of a basal pressure might be considered after preparation similar to that used for basal metabolism. It should be determined 10 to 12 hours after the last meal of the previous night and after resting for half an hour in a warmed room."

Alam and Smirk (1938) indicated that they were using a basal blood pressure as a base-line in studies of blood pressure raising reflexes and later (1943) obtained results approximating to the basal blood pressure with a procedure which they termed emotional desensitization to the presence of the observer and to the process, of sphygmomanometry, with special attention to rest in a warm quiet room, but taking no special precautions as to the time of day or the time after the last meal. Alam and Smirk, from observations of blood pressure obtained by this method, suggested that readings obtained under ordinary clinical conditions should be called casual blood pressures, and those obtained under basal conditions, basal blood pressures; and that the difference between casual and basal blood pressure levels should be called the supplemental blood pressure. This represents the degree of elevation of the blood pressure above the basal level due to physical, metabolic, mental, and emotional stimuli at the time the casual blood pressure is taken. The less the physical metabolic and emotional pressor stimuli at the time the casual pressure is observed the nearer will this pressure approach the basal level for that individual and the lower will be the supplemental pressure.

Later Smirk (1945) showed that the technique Alam and he had described for basal blood pressure determination, when applied without regard to the time of the previous meal, produced lower results than were ordinarily obtained by the method advocated by the Cardiac Societies. He showed further that when both methods were combined the resulting basal blood pressure level was slightly lower than with either method when used alone.

This investigation was primarily designed to determine if in health and in essential hypertension the basal pressure is in fact a constant measurement for the individual. In the course of the work observations were made to investigate a more rapid and convenient method of basal blood pressure 
determination using a hypnotic drug. Different degrees of variability of blood pressure were observed among the hypertensive subjects studied and an analysis of the supplemental pressure of these and of normal subjects was undertaken to determine if there was also a relationship between the presence of congestive heart failure and variability of the blood pressure level.

\section{METHOD}

Except in the group of subjects in which an hypnotic drug was given the method of basal blood pressure determination which was used throughout this study was that outlined by Smirk (1945), namely a combination of the routine described by the combined committee on blood pressure measurement and that of Alam and Smirk (1943). The procedure was designed to measure the blood pressure when the subject was in the basal metabolic state and was free from states of emotion or mental alertness.

Many subjects will not attain a basal state if they gain the impression that the blood pressure measurements are merely the prelude to some unfamiliar and possibly unpleasant procedure. For this reason the subjects were informed on the day before the determination that no procedure would take place except blood pressure measurements with which they were already familiar. The object of the experiment and the procedure to be used were outlined and instruction was given to keep the mind blank and the body relaxed and still, as though trying to go to sleep. Talking was not allowed. In the morning the subject used a urinal or bed pan, if necessary, soon after waking. The subject did not get out of bed nor have any food but was moved to a quiet warm single room where he or she remained alone, without reading and, if possible, asleep for about half an hour. The observer then entered quietly, adjusted the sphygmomanometer and proceeded to record the level of the blood pressure about twice each minute, for the next twenty to thirty minutes. No other person entered the room during the determination. The observer did not move about the room or engage in any pursuits that would divert the subject's attention. The lowest systolic pressure which was recorded three times and the accompanying diastolic pressure were taken as the basal blood pressure.

All the blood pressure measurements were made on the left arm which was supported at heart level. The same mercury manometer was employed throughout the study using the method of judging systolic and diastolic level detailed by the British and American Cardiac Societies. All the observa- tions were made by the author and the sphygmomanometer used was checked against a standard instrument several times in the course of the study. In each of the subjects casual blood pressure readings were taken on the day before or later on during the day on which basal blood pressure determinations were made. These casual blood pressure measurements were designed to conform with those ordinarily made in wards or clinic. The measurements were all made with the patient reclining and on the left arm which was supported at heart level, but no special precautions were taken to eliminate the effects of exercise, meals, or emotional factors.

\section{SUBJeCTS}

The subjects upon whom measurements were made were mainly convalescent patients awaiting discharge. These subjects had blood pressures ranging from normal to hypertensive levels. The reason for admission to hospital varied, most of the subjects having complaints quite distinct from essential hypertension such as hernia, peptic ulcer, chronic diarrhœa, etc., but there were several whose main complaint was associated with essential hypertension, such as headaches, dizziness, encephalopathy, or hemiplegia.

To assess the degree of constancy of casual and basal blood pressure levels, casual blood pressure readings were made and the procedure of determining the basal blood pressure was carried out from four to seven times in each of 33 subjects. These subjects were grouped according to the level of the casual blood pressure.

Group I. Eleven subjects in whom the mean casual systolic blood pressure was $132 \mathrm{~mm}$. of mercury or less and in whom no single systolic reading exceeded 140 . The casual diastolic readings were never above 90.

Group II. Eight subjects in whom the casual blood pressures were higher than those of Group I but whose mean casual systolic pressure did not exceed 160: the mean casual diastolic readings were all below 90 .

Group III. Fourteen subjects with essential hypertension without albuminuria but including some with early congestive heart failure, whose mean casual systolic pressures all exceeded 160 and whose mean diastolic pressures were all above 90.

The effect of premedication with pentobarbitone soluble in decreasing the time required for basal blood pressure determination was studied in nine subjects equally distributed among the above three groups.

In 44 subjects casual blood pressure levels were measured and basal blood pressure determinations 
were carried out on one occasion and the supplemental (casual minus basal) pressure determined by calculation. These subjects were grouped as before into a group of 14 subjects with normal levels of casual blood pressure and a group of 8 subjects with intermediate casual blood pressure levels but the third group of hypertensive subjects was divided into two sections. Group III A: 13 hypertensive subjects whose blood pressures conformed to the levels outlined previously for Group III in general but without history or signs of congestive heart failure. Group III B: 12 hypertensive subjects as above except that they showed signs of congestive heart failure.

\section{Results AND Discussion}

To determine the variability from day to day of both casual and basal pressures the results in the first series of 33 subjects were graphed separately (Systolic pressures Fig. 1 to 6). The graphs were drawn on a common chart for each group so that the zero line represents the mean systolic blood pressure of each subject and each continuous line shows the fluctuation of that subject's blood pressure above and below the zero line from day to day. The charts were all drawn to the same scale.

Fig. 1 shows the variation among the casual systolic blood pressures measured from day to day in each of 11 normal subjects (Group I) while Fig. 2 shows the variation of the basal systolic pressures in the same subjects.

The close approximation of the lines in Fig. 2 suggests that over the period of two weeks to one month during which observations were made the basal systolic pressure of these subjects was almost constant. The greater degree of variability in the casual blood pressure is shown by the wider spread of the lines in Fig. 1 than in Fig. 2.

The charts of Group II-Fig. 3 and 4-show an even more marked difference between the variation of casual and basal systolic pressures. This group of subjects was selected to conform to the prehypertensive subjects of Hines (1940) and the hyper-reactors of Ayman (1934). In this group the casual systolic pressures (Fig. 3) are more variable than those of the normal subjects of Group I (Fig. 1). The basal pressures of this group seem to be slightly less constant than in the normal subjects. There is a general tendency for the first basal pressure in each subject to be high. This initial high reading may indicate that the first attempt was unsuccessful owing to the presence of concealed emotional tension or of apprehension. When these doubtful initial readings are eliminated from the charts the basal pressures upon which reliance may be placed are seen to be almost constant.

Fig. 5 and 6 show the variability of casual and basal systolic pressures in the hypertensive subjects of Group III. The gross variation of the casual pressures from day to day (Fig. 5) is striking, while the basal pressures (Fig. 6) are less variable and, if the first observation is excluded for the reasons given above, the remainder show appreciably less variation than do the casual pressures.

The degree of constancy shown by the basal systolic figures in general and in Group $I$ in particular (Fig. 2), increases in significance when the following points are considered. It had been shown that with repetition of the readings, blood pressure levels tend to drop due to the removal of some of the emotional stimulation or mental alertness associated with sphygmomanometry. Such repeated readings, would, therefore, tend to become more constant. This factor plays a large part in producing the relatively small variation of casual pressure seen in Fig. 1, as the subjects were having readings taken over many days, and towards the end of the series they were so accustomed to the procedure that the casual pressure would fall and the readings observed would show some degree of false constancy not actually to be expected in a series of truly casual readings. The basal blood pressures observed, on the other hand, were readings taken after placing the subject as far as possible in basal conditions. The experimental errors which could occur would all tend to give readings rather higher than the true basal level, and such errors might be many. Small, apparently innocuous variations of technique might occur. On one occasion it was found that two subjects were showing rather higher basal pressures than had previously been recorded. On investigation it was found the subjects had walked from the ward to the room where the basal pressures were measured instead of being moved in bed. These readings were, of course, discarded as being non-basal. One patient, who was suffering from peptic ulceration, showed a high level of basal pressure after a night disturbed by slight but annoying abdominal pain.

The most remarkable divergences from the usual basal level were shown when concealed emotional factors were present. One hypertensive subject had received by letter disturbing news about her son's conduct. The basal pressure next morning was $50 \mathrm{~mm}$. higher than usual and only after prolonged questioning was the cause revealed. In another instance the subject, a soldier, showed slowly increasing levels of basal pressure. Interrogation failed to reveal any cause for anxiety known to the subject, but it was found by chance that he was 

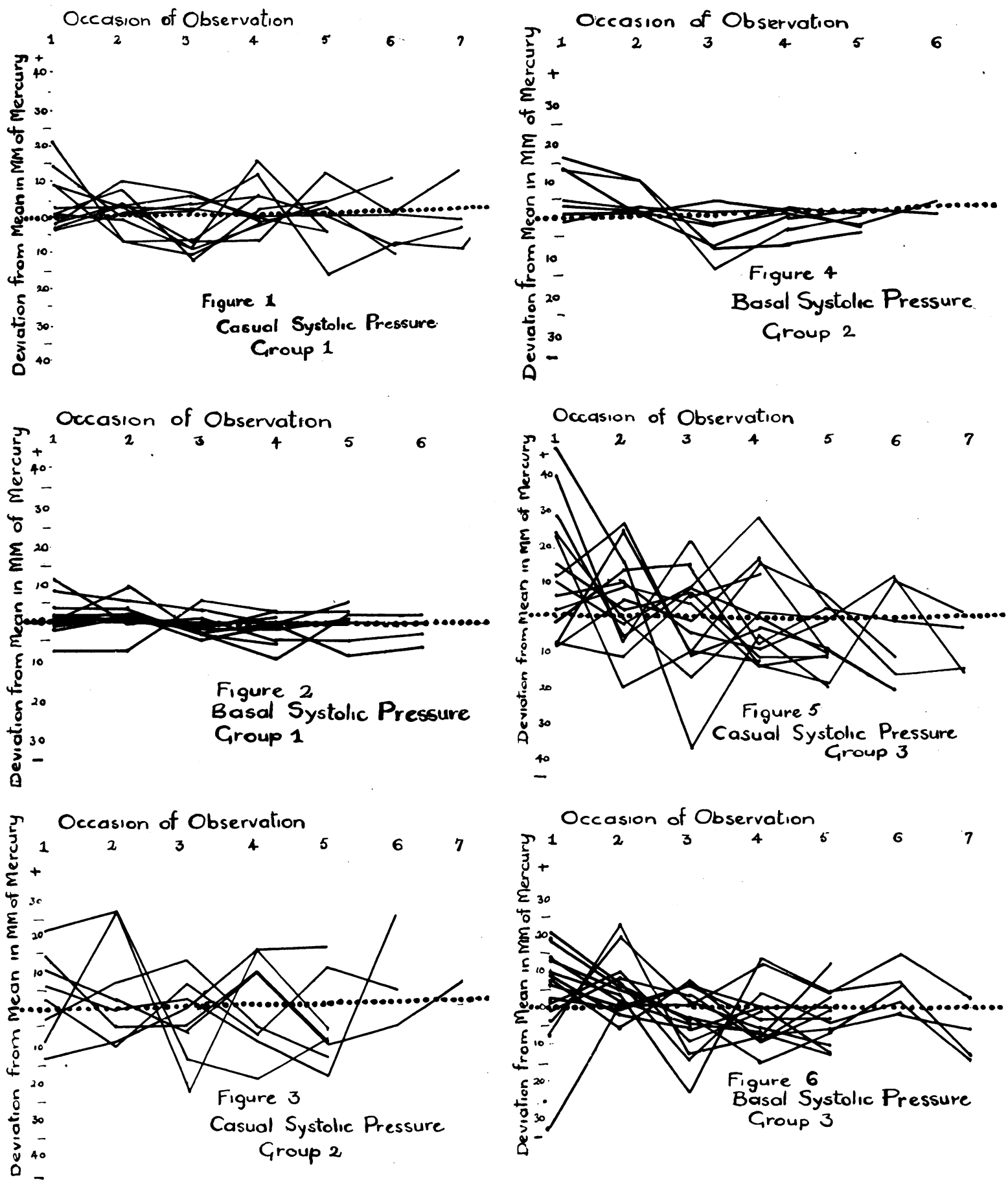

FIG. 1-6.-The degree of variation from day to day of the systolic blood pressure levels of the subjects in the three groups. The levels of the individual readings of each subject are plotted as points above or below a zero line that represents the mean level of that subject. The points are connected by lines so that each line shows the variation of blood pressure in one subject. The casual and basal pressures are shown separately. In each group the lines for the individual subjects are drawn to the same scale on the one chart so that the one common zero line represents the mean of each subject.

The degree of variation is shown by the extent of spread of the lines above and below the zero line. The scale is in millimetres of mercury.

The subjects were grouped as follows: Group I, normal blood pressure levels; Group II, intermediate pressure levels; Group III, hypertensive levels. 

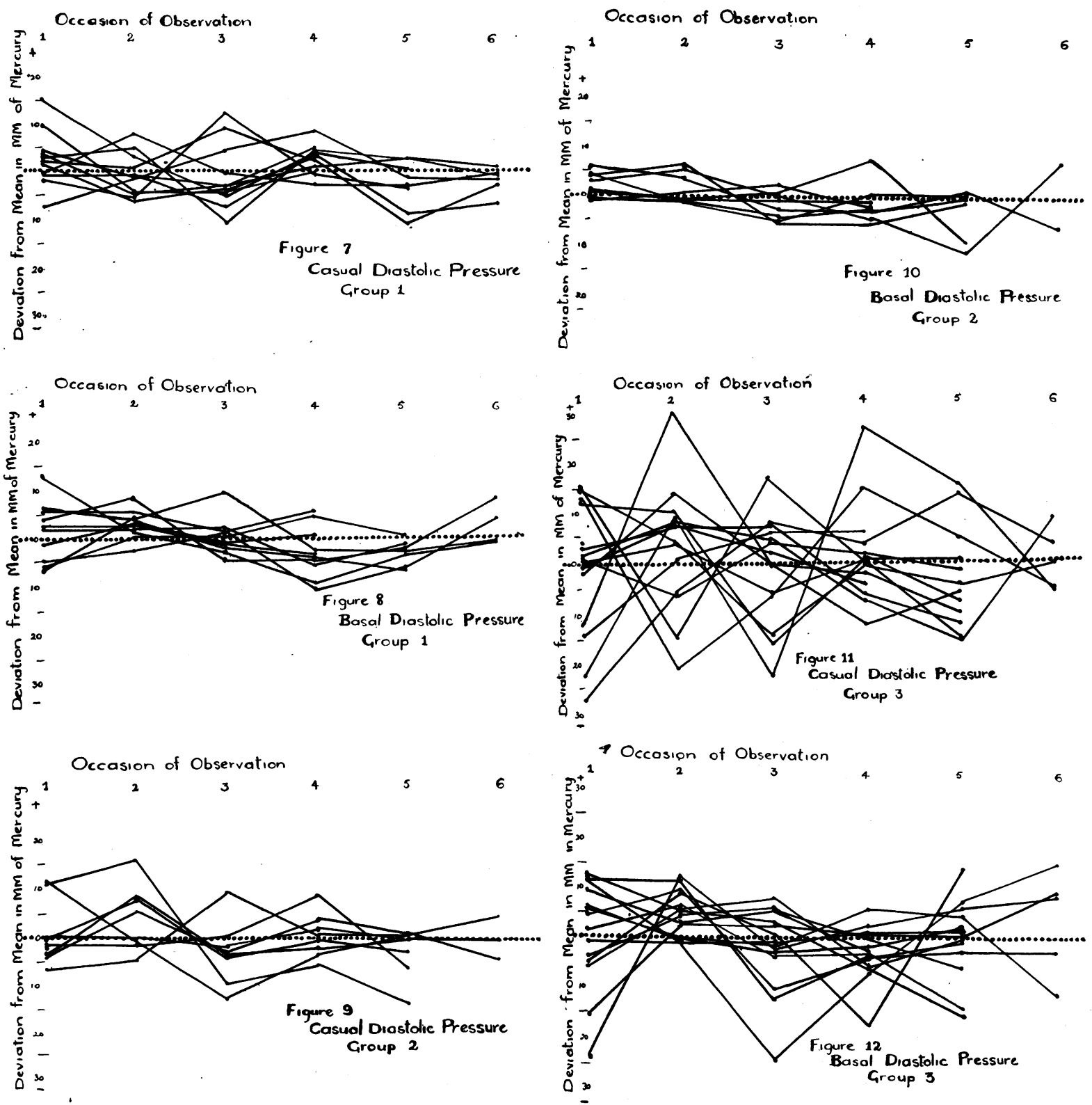

Fig. 7-12.-The degree of variation from day to day of the diastolic blood pressure levels of the subjects in the three groups. The casual and basal pressures are shown separately. For details see Fig. 1-6.

due to have an Army Reboard to decide if his military service would be terminated. He was discharged from the Army, as he had hoped, and further basal blood pressure readings returned to their previous level. In such cases there was failure to obtain a basal pressure. Some such errors may have been present without our knowledge in a few of the readings which have been accepted as basal and would tend to make the variation observed in the basal pressure appear to be greater than the true variation, which must be slight.

One of the most constant experimental errors which must be considered in assessing the accuracy of the results is that due to the sphygmomanometer. This error is due to the vibration of the mercury column with the pulse wave and to a few millimetres 
rise and fall of the blood pressure during respiration. Neither of these factors is constantly present but both may, in certain subjects, be the source of a relatively large error. There is also the factor of personal error which is more important in assessing the diastolic level. A reasonable estimate of the total possible error would be $\pm 4 \mathrm{~mm}$. of mercury for the systolic and $\pm 8 \mathrm{~mm}$. of mercury for the diastolic. Larger errors were prevented by strict adherence to the method of blood pressure measurement outlined by the combined committee of the Cardiac Societies.

When these sources of error are kept in mind and Fig. 1 and 2 reconsidered it is obvious that the basal systolic pressure must be considered to be constant from day to day in normal subjects in a physiologically constant state. Fig. 1 to 6 show that casual systolic readings are much more variable in essential hypertension than in health. Throughout the series of observations it was noticed that hypertensive patients did not relax easily, neither physically nor mentally. Basal levels of blood pressure were more difficult to attain especially if there was at the time any emotional disturbance such as apprehension. During basal blood pressure determination sudden noises, interruptions from the corridor, lift-gates slamming, etc., would produce larger elevations of pressure in hypertensives than comparable stimuli in normal subjects. It has previously been noted that definite emotional pressor factors were found in some hypertensives and that these factors were difficult to eliminate. Some hypertensives may be inherently emotional, tense and restless. Others, no doubt, have vascular systems which are more reactive than the average to emotional or other stimuli. Very likely still others are inherently emotional and have, as well, a high vascular reactivity.

In any experimental studies to determine the degree of improvement in essential hypertension, a comparison of the basal blood pressure levels before and after treatment would give a more accurate measure of the clinical result than would a comparison of casual levels, which are so highly variable. In almost all reports on the results of therapy in essential hypertension conclusions have been drawn from an observed difference in casual blood pressure levels and in many the difference observed could easily have been due to chance variation.

The levels of the diastolic pressures were also recorded throughout the study and the results graphed as for the systolic pressures. Fig. 7 and 8 show the variation of casual and basal diastolic pressures of the normal subjects (Group I), Fig. 9 and 10 the casual and basal diastolic pressures of Group II with rather higher pressure levels, and
Fig. 11 and 12 show the casual and basal diastolic pressures of the hypertensive subjects (Group III). The same general features may be seen as with systolic pressures namely that the basal levels are much more constant than are the casual levels. Fig. 8 shows that within the rather larger limit of experimental error $( \pm 8 \mathrm{~mm}$. of mercury) the basal diastolic pressure is constant from day to day in healthy subjects.

It is often stated that the diastolic pressure is less variable than the systolic pressure. Except in the subjects with slightly abnormal blood pressure levels (Group II-Fig. 3 and 4, 9 and 10) this does not seem to apply to the subjects studied as the spread of the graphs in Fig. 7 and 11 is not significantly less than the spread in Fig. 1 and 5 respectively. It is to be expected that in the hyper-reactors of Group II, the diastolic pressures, both casual and basal, would be more constant than the systolic pressures because lability of the systolic pressure is the main criterion of selection in this group.

The levels of blood pressure observed in this section of the study are shown in Fig. 13. For Group I, the subjects with normal blood pressure level, the mean casual pressure was $118 \mathrm{~mm}$. systolic and 79 diastolic, while the mean basal pressure was 103 systolic and 72 diastolic. In Group II, composed of prehypertensive subjects, the mean casual level was of course higher, namely $138 \mathrm{~mm}$. systolic and $82 \mathrm{~mm}$. diastolic, while the mean basal level was not appreciably different from those of group one, namely $109 \mathrm{~mm}$. systolic and $69 \mathrm{~mm}$. diastolic. In the third group, in which hypertensive subjects of varying levels were classed, the mean casual pressure was $177 \mathrm{~mm}$. systolic and $117 \mathrm{~mm}$. diastolic. It should be emphasized here that in any group of subjects the mean supplemental pressure must be calculated from the individual casual and basal levels of each subject. The difference between the mean casual level, and the mean basal level gives no information as to the mean supplemental pressure of the group.

In several cases in each group of subjects 1.5 grains of sodium pentobarbitone was given by mouth shortly after waking on the morning of the basal blood pressure determination. It was found that basal levels were more quickly attained in this way, in nine of the cases in whom an average of over eight minutes was required for the blood pressure to reach basal levels without premedication, the use of the drug gave equivalent levels in under three minutes. For accurate work, however, a much longer time should be spent in the determination because in some cases low levels are attained only after twenty to thirty minutes of habituation. The hypnotic had the added advantage 


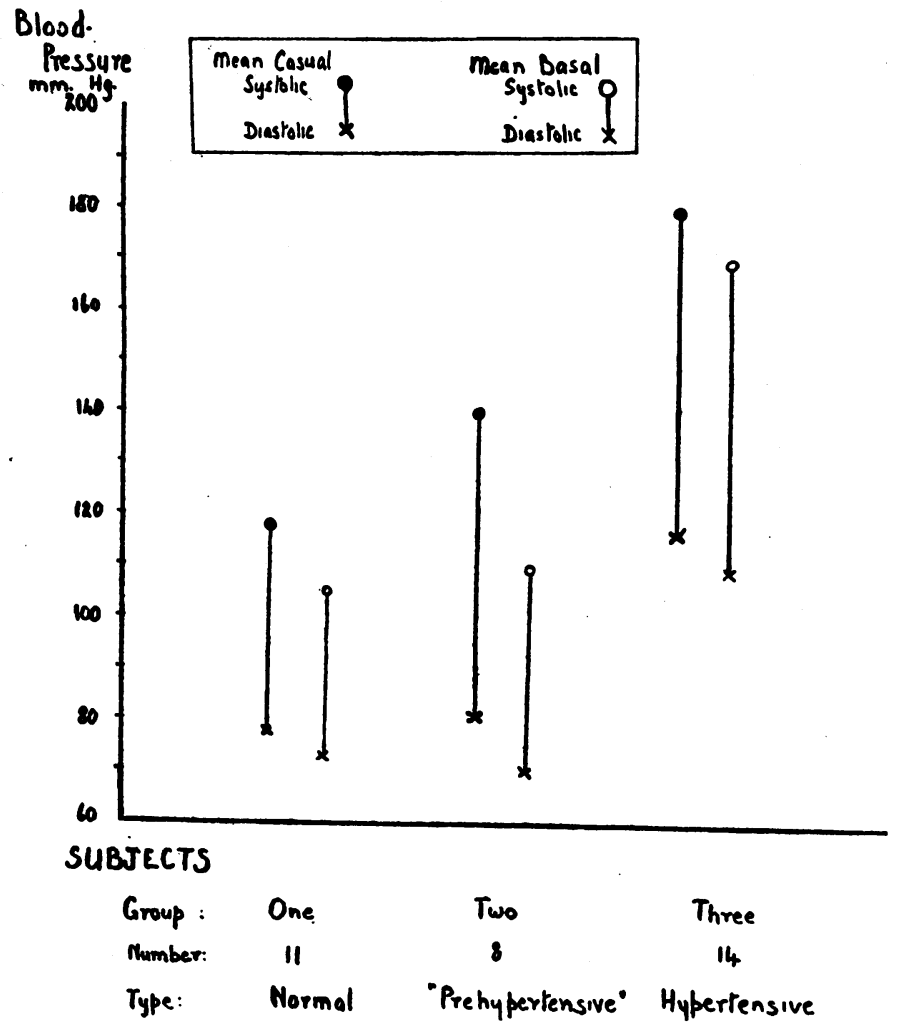

Fig. 13. - The mean casual and basal blood pressure levels in Groups I, II, and III.
The Supplemental Pressure

In the course of the investigation there were found to be striking differences in the drop in blood pressure observed in different subjects during basal blood pressure determination. To amplify this observation a further series of 44 subjects was studied, one casual blood pressure reading and one basal blood pressure determination being carried out on each subject and the supplemental pressure determined by subtraction. The supplemental pressure is a measure of the degree of elevation of the blood pressure due to emotional, nervous, and metabolic factors and to physical exertion and therefore varies from person to person and from moment to moment but gives an indication of the reactivity of the blood pressure.

The subjects were grouped as before into three main groups according to the level of the blood pressure, normal, intermediate, and hypertensive, but the hypertensive group was further subdivided into two sections. Group III A consisted of 13 hypertensive subjects without signs or symptoms of congestive heart failure and Group III B was composed of 12 subjects with vary-

of keeping the patient rested and quiet for a much longer period so that the determination of the basal blood pressure level could be made at a time much more convenient for the investigator than soon after the subject wakes in the morning as was originally proposed.

The method of basal blood pressure determination used in this study was designed to give as accurate an estimate of the true basal blood pressure as it is possible to obtain. In practice a fair approximation may be obtained on outpatients in the consulting room by a trained assistant with preparation of the patient as for basal metabolic rate determination. The accuracy of such readings would be increased if premedication with a rapidly acting barbiturate, such as sodium pentobarbitone as described above, were used and if several determinations were made on different occasions as the first reading is uspally higher than later ones. The effect of the premedication rapidly wears off, all of the subjects studied being quite able to carry on their daily work two hours after the test. ing degree of congestive heart failure.

Table I shows the results of the investigation in these 44 subjects. There are obvious and striking differences in the mean supplemental pressures of the various groups. The normal subjects showed supplemental pressures of about $20 \mathrm{~mm}$., while the group with intermediate casual pressure levels showed a rather higher figure as was to be expected from the method of selection. In the subjects with essential hypertension the mean supplemental pressure was over twice that of the normals. When the essential hypertension was complicated by congestive heart failure, however, the supplemental pressure was barely one-fifth of that in the uncomplicated cases and barely half that of the normal subjects.

To determine the significance of these observations a statistical analysis was made, the results (Table I) giving mean supplemental pressures in the three groups. To assess the statistical significance of the differences observed between these mean values of supplemental pressure, the standard error of each 
TABLE I

The Mean. Supplemental Blood Pressure and Statistical Summary

\begin{tabular}{|c|c|c|c|c|c|}
\hline \multirow[t]{2}{*}{ Group and number of subjects } & \multirow[t]{2}{*}{$\begin{array}{l}\text { Mean supple- } \\
\text { mental pressure } \\
\text { in millimetres } \\
\text { of mercury }\end{array}$} & \multirow[t]{2}{*}{$\begin{array}{c}\text { Standard } \\
\text { deviation in } \\
\text { millimetres of } \\
\text { mercury }\end{array}$} & \multicolumn{3}{|c|}{$\begin{array}{l}\text { Difference between mean supplemental } \\
\text { pressures with standard error of difference } \\
\text { in millimetres of mercury }\end{array}$} \\
\hline & & & $\begin{array}{l}\text { Groups } \\
\text { compared }\end{array}$ & $\begin{array}{c}\text { Difference } \\
\text { with standard } \\
\text { error of } \\
\text { difference }\end{array}$ & Significance \\
\hline I. 14 normals $\quad . . \quad$.. & $18 \cdot 2$ & $8 \cdot 51$ & $\begin{array}{l}\text { I }(18 \cdot 2) \\
\text { II }(25 \cdot 0)\end{array}$ & $6 \cdot 8 \pm 4 \cdot 97$ & Nil \\
\hline II. 8 prehypertensives $\quad . . \quad \ldots$ & $25 \cdot 0$ & $12 \cdot 51$ & $\begin{array}{l}\text { III A }(44 \cdot 6) \\
\text { I }(18 \cdot 2)\end{array}$ & $26 \cdot 4 \pm 4 \cdot 27$ & High \\
\hline 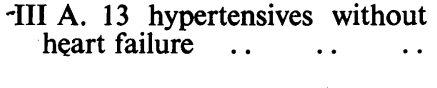 & $44 \cdot 6$ & $13 \cdot 02$ & $\begin{array}{l}\text { III A }(44 \cdot 6) \\
\text { III B }(9 \cdot 0)\end{array}$ & $35 \cdot 6 \pm 3 \cdot 82$ & Very high \\
\hline $\begin{array}{l}\text { III B. } 12 \text { hypertensives } \\
\text { failure } \\
\begin{array}{lllll}. . & . . & .\end{array}\end{array}$ & $9 \cdot 0$ & $4 \cdot 32$ & $\begin{array}{l}\text { I }(18 \cdot 2) \\
\text { III B (9·0) }\end{array}$ & $9 \cdot 2 \pm 2 \cdot 59$ & High \\
\hline
\end{tabular}

difference was calculated. A statistically significant difference between two mean values exists when the numerical difference between the values exceeds double the standard error of that difference. In this table it may be seen that the supplemental pressure of the group of hypertensive subjects is significantly higher than that of either the normal subjects or the subjects with hypertension complicated by heart failure, and the supplemental pressure of this latter group is significantly lower than that of the normal subjects.

The great elevation of the supplemental pressure in essential hypertension accords with the previous observation that in essential hypertension the blood pressure shows marked variation. The low supplemental pressure in essential hypertension with congestive heart failure shows that the reactivity of the circulatory system to metabolic factors, physical work, and nervous and emotional factors is reduced. It might be argued that the basal blood pressure in these subjects would therefore be constant. In those subjects on whom repeated basal blood pressure determinations were made, however, it was found that from day to day the level of the basal blood pressure was not quite constant although during each determination the level of the systolic pressure changed very little. This suggests that a possible explanation for the low supplemental pressure found in these heart failure cases is that the method of basal blood pressure determination does not succeed in removing the pressor factors mentioned above and that a true basal level is not attained. Possible causes for this are the frequent presence of dyspnœa and Cheyne-Stokes respiration and the general discomfort of the patient, all of which would tend to prevent the attainment of completely basal conditions. In several patients, however, determinations were made during both the acute illness when dyspnœa and general discomfort were marked and during convalescence when the patient was well. In these cases the supplemental pressure during convalescence was as low as that found during the acute phase of heart failure.

Apart from this effect the strictly controlled conditions of the experiment do not seem to admit of other reasons for failure to attain a basal level and there is, therefore, no reason to suspect that the process of basal blood pressure determination was any less effective in the patients with congestive heart failure than in those with uncomplicated essential hypertension. Similarly, the presence of the low supplemental pressure in the convalescent patient suggests that heart failure by itself is not sufficient to account for the vastly different supplemental pressures found in compensated and in failed essential hypertension. The reduction of cardiac output which may accompany congestive heart failure may account, in part, for the low supplemental pressure observed. For in cases of congestive heart failure the cardiac output is close to its maximum and cannot be much increased in response to the usual pressor stimuli. The blood pressure, which is maintained by the cardiac output and the peripheral resistance, may, however, be altered by variation in the latter factor. 
If alteration in the cardiac output was the sole cause of the low supplemental pressure one would expect that with recovery of some degree of circulatory compensation the low supplemental pressure observed in the acute phases would be expected to rise. In the cases observed this did not occur.

It is possible that the low supplemental pressure observed in congestive heart failure was present before failure occurred and that it is a manifestation of reduced variability of the peripheral resistance rather than reduced variability of the cardiac output. A low supplemental pressure may indeed indicate an early onset of congestive heart failure, in that with a sustained high level of peripheral resistance the cardiovascular system would fail sooner than when, with the same level of casual blood pressure and a high supplemental pressure, the cardiovascular system was relieved, during rest, of a large part of the peripheral resistance. The presence of a low supplemental pressure in cases of essential hypertension may, therefore, be of assistance, along with the degree of hypertension, in assessing prognosis, especially as to the onset of congestive heart failure.

The hypertension of chronic nephritis, in which only a small amount of variation occurs (Kylin 1921; Ayman 1931; Gatman, Nassif Amin, and Smirk 1943) is, in this respect distinctly different from the hypertension of subjects with early stages of essential hypertension (Group III A) in which a large supplemental pressure occurs. On the other hand the hypertension of chronic nephritis, which is widely accepted as being mediated by the renin system, resembles that of the failed cases of essential hypertension of Group III B in that a low supplemental pressure occurs in both. The observation that the relief of renal congestion by an improvement in cardiovascular function does not increase the supplemental pressure suggests that renal congestion by itself is not sufficient to account for the similarity of the blood pressure responses of chronic nephritis and of cases of failed essential hypertension.

\section{Conclusions}

The basal blood pressure of normal subjects is constant for periods of one to three weeks.

The casual blood pressure of normal subjects is a variable figure.

The casual blood pressure of subjects with essential hypertension is exceedingly variable.

The basal blood pressure of subjects with essential hypertension is much more constant than the casual blood pressure. A part of this variability is probably due to the greater difficulty in securing basal condition in these subjects.

The use of an hypnotic drug (soluble pentobarbitone, 1.5 grains given by mouth half to one hour before the test) preparatory to basal blood pressure determination gives levels of basal blood pressure comparable to those obtained without such premedication and greatly reduces the time taken to obtain a basal level in nearly all cases.

The mean supplemental pressure in a group of normal subjects was $18 \mathrm{~mm}$. of mercury. In a group of hypertensive subjects the mean supplemental pressure was $44 \mathrm{~mm}$. of mercury. The difference between these figures is statistically significant. In a group of hypertensive subjects with congestive heart failure, however, the mean supplement pressure was $9 \mathrm{~mm}$. of mercury, significantly lower than that of the normal subjects and, even more 'so, of the hypertensive subjects without heart failure.

Thanks are due to Professor F. H. Smirk for his encouragement, advice, and criticism throughout the preparation of this paper, and to Miss Walsh for clerical assistance.

Part of the investigation was aided by a grant from the Dunbar Research Fund of the University of Otago for which I wish to thank the University Council.

\section{REFERENCES}

Addis, T. (1922). Arch. intern. Med., 29, 539. Alam, G. M., and Smirk, F. H. (1938). Clin. Sci., 3, 259.

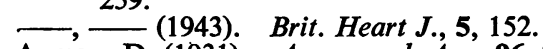

Ayman, D. (1931). Amer. med. Ass., 96, 2091.

(1934). Arch. intern. Med., 53, 792.

Hines, E. A. (1940). J. Amer. med. Ass., 115, 271.
Gatman, M., Nassif Amin and Smirk, F. H. (1943). Brit. Heart J., 5, 161.

Kylin, E. (1921). Acta. med. Scand., 55, 368.

Report of the Combined Committees of the Cardiac Society of Great Britain and Ireland and the American Heart Association (1938). J. Amer. med. Ass., 133, 294.

Smirk, F. H. (1945). Brit. Heart J., 6, 176. 